\title{
ON THE FIRST PONTRYAGIN FORM OF A SURFACE
}

\author{
ROBERTO FERREIRO PÉREZ and JAIME MUÑOZ MASQUÉ
}

Communicated by Karl-Hermann Neeb

\begin{abstract}
The first Pontryagin form of a compact orientable surface $M$ determines a canonical pre-symplectic structure on the space of Riemannian metrics on $M$. The first equivariant Pontryagin form determines a canonical moment map for it. We study the corresponding symplectic reduction and we state (Theorem 6) that the symplectic quotient is the Teichmüller space of the surface with the Weil-Petersson symplectic form.
\end{abstract}

\section{Introduction}

The universal Pontryagin forms are differential forms on the first jet-bundle of the bundle of Riemannian metrics of a manifold $M$. They are closed and invariant under the natural action of the diffeomorphism group of $M$. Pulling-back a universal Pontryagin form by means of the first jet prolongation $j^{1} g$ of a Riemmanian metric $g$ we obtain a closed differential form on $M$. As the space of Riemannian metrics is contractible, the maps $j^{1} g$ are homotopic for different metrics $g$, and hence the cohomology class of the form obtained on $M$ is independent of the metric $g$ chosen. This cohomology class is the corresponding Pontryagin class of $M$. In this way we recover the result that the Pontryagin classes are independent of the Riemannian metric used to construct them.

Moreover, with this construction we obtain more than merely the Pontryagin classes. The key point is the fact that there are non-zero universal Pontryagin forms of degree greater than the dimension of $M$. We show that these higherorder universal Pontryagin forms can be interpreted geometrically. Concretely, we study the interpretation of the first universal Pontryagin form of a compact and oriented surface. In addition, the equivariant Pontryagin forms are also introduced, thus providing canonical equivariant extensions of the universal Pontryagin forms.

By applying the results of [6] to the case of Riemannian metrics we obtain that the first universal Pontryagin form of a compact and oriented surface determines a 
pre-symplectic structure on the space of Riemannian metrics. The first equivariant Pontryagin form determines an equivariant extension of this form, that is, a moment map for it. We study the corresponding symplectic reduction, and the result is that the Marsden-Weinstein quotient is the Teichmüller space of the surface, and hence we obtain a natural pre-symplectic structure on it. Finally we state that this pre-symplectic structure coincides (up to an scalar factor) with the symplectic form corresponding to the Weil-Petersson metric.

This result is the analog of the result proved in [6, Example 19] according to which the second Chern form of a principal SU(2)-bundle over a compact Riemann surface determines the natural symplectic structure on the space of connections, and the second equivariant Chern form determines the natural moment map for it, both of them introduced in [1], where they are used to determine the natural symplectic structure on the moduli space of flat connections.

\section{The Universal and Equivariant Pontryagin Forms}

Let $M$ be an oriented, compact, connected $n$-dimensional manifold without boundary, let $q: \mathcal{M}_{M} \rightarrow M$ be its bundle of Riemannian metrics, let $q_{1}: J^{1} \mathcal{M}_{M} \rightarrow M$ be its first jet bundle, and let $\operatorname{Met} M=\Gamma\left(M, \mathcal{M}_{M}\right)$ denote the space of Riemannian metrics on $M$.

Let Diff ${ }^{+} M \subset \operatorname{Diff} M$ be the subgroup of orientation-preserving diffeomorphisms. We set $\mathcal{G}=\operatorname{Diff} M \times \mathbb{R}$ and $\mathcal{G}^{+}=\operatorname{Diff}^{+} M \times \mathbb{R}$.

The group $\mathcal{G}$ acts on the bundle of metrics $\mathcal{M}_{M}$ in the following way. The natural lift of a diffeomorphism $\phi \in \operatorname{Diff} M$ to the bundle of metrics $\bar{\phi}: \mathcal{M}_{M} \rightarrow \mathcal{M}_{M}$ is defined by $\bar{\phi}\left(g_{x}\right)=\left(\phi^{*}\right)^{-1}\left(g_{x}\right) \in\left(\mathcal{M}_{M}\right)_{\phi(x)}, \phi^{*}: S^{2} T_{\phi(x)}^{*} M \rightarrow S^{2} T_{x}^{*} M$ being the induced homomorphism. Hence, we have $q \circ \bar{\phi}=\phi \circ q$, and then, we define $(\phi, t) \cdot g_{x}=\exp (t) \bar{\phi}\left(g_{x}\right)$, for $(\phi, t) \in \mathcal{G}, g_{x} \in \mathcal{M}_{M}$. This action of $\mathcal{G}$ on $\mathcal{M}_{M}$ induces a prolonged action of $\mathcal{G}$ on $J^{1} \mathcal{M}_{M}$ in a natural way.

The basic idea of this section is to consider the classical construction of Pontryagin classes, but applying it to $J^{1} \mathcal{M}_{M}$ instead of to $M$ itself.

Let $\pi: F M \rightarrow M$ be the linear frame bundle of $M$. The group $\mathcal{G}$ acts on $F M$ by setting $\tilde{\phi}: F M \rightarrow F M, \tilde{\phi}\left(u_{x}\right)=\phi_{*}\left(u_{x}\right)$ for $\phi \in \operatorname{Diff} M$, and $(\phi, t) \cdot u_{x}=$ $\exp (-t / 2) \tilde{\phi}\left(u_{x}\right)$ for $(\phi, t) \in \mathcal{G}, u_{x} \in F M$.

The pull-back bundle $q_{1}^{*} F M \rightarrow J^{1} \mathcal{M}_{M}$ is a principal $\operatorname{GL}(n, \mathbb{R})$-bundle. The actions of $\mathcal{G}$ on $\mathcal{M}_{M}$ and $F M$ induce an action of $\mathcal{G}$ on $q_{1}^{*} F M$. We consider the subbundle $O M=\left\{\left(j_{x}^{1} g, u_{x}\right) \in q_{1}^{*} F M ; u_{x}\right.$ is $g_{x}$-orthonormal $\}$. Then, $O M \rightarrow$ 
$J^{1} \mathcal{M}_{M}$ is a principal $\mathrm{O}(n)$-bundle, which admits a canonical connection:

Theorem 1. There exists a unique $\mathcal{G}$-invariant connection form $\boldsymbol{\omega}$ on the bundle $O M \rightarrow J^{1} \mathcal{M}_{M}$.

This connection is called the universal Levi-Civita connection and its curvature form is denoted by $\Omega$.

Let us recall how this Levi-Civita connection is defined. A point in $j_{x}^{1} g \in J^{1} \mathcal{M}_{M}$ consist on a point $x \in M$, a metric $g(x)$ on $T M$, and the first derivatives $\partial g(x) / \partial x^{i}$ of $g$ at $x$. As the Levi-Civita connection of a metric depends on the first derivatives of the metric, by collecting the corresponding connections at the points of $q_{1}^{*} F M$ we obtain a connection form $\boldsymbol{\omega}_{\text {hor }}$ on $q_{1}^{*} F M \rightarrow J^{1} \mathcal{M}_{M}$; Precisely, $\boldsymbol{\omega}_{\text {hor }}(X)=$ $\omega^{g}\left(\left(\bar{q}_{1}\right)_{*} X\right)$, for each $X \in T_{\left(j_{x}^{1} g, u_{x}\right)} q_{1}^{*} F M$, where $\bar{q}_{1}: q_{1}^{*} F M \rightarrow F M$ is the natural projection and $\omega^{g}$ denotes the Levi-Civita connection form of $g$. That the connection $\boldsymbol{\omega}_{\text {hor }}$ obtained in this way is $\operatorname{Diff} M$-invariant follows from the fact that the Levi-Civita connection is natural with respect to the action of Diff $M$ on the space of Riemannian metrics (e.g. see [5]). However $\boldsymbol{\omega}_{\text {hor }}$ is not a Riemannian connection, i.e., is not reducible to $O M$. In [7] it is show that this connection can be modified by adding to $\boldsymbol{\omega}_{\text {hor }}$ a term depending on the contact structure of $J^{1} \mathcal{M}_{M}$ in such a way that the connection $\boldsymbol{\omega}$ obtained is $\operatorname{Diff} M$-invariant and Riemannian.

The universal $k$-th Pontryagin form of $M, p_{k}(\boldsymbol{\Omega}) \in \Omega^{4 k}\left(J^{1} \mathcal{M}_{M}\right)$ is defined to be the form obtained by evaluating the $k$-th Pontryagin polynomial at the curvature $\boldsymbol{\Omega}$ of $\boldsymbol{\omega}$ according to the Chern-Weil theory of characteristic classes (e.g. see [9, Chapter XII]).

Theorem 2. The universal Pontryagin forms are closed, $\mathcal{G}$-invariant and satisfy the following universal property: For every Riemannian metric $g$ on $M$ we have $\left(j^{1} g\right)^{*}\left(p_{k}(\boldsymbol{\Omega})\right)=p_{k}\left(\Omega^{g}\right)$, where $\Omega^{g}$ is the curvature form of $g$.

The Pontryagin forms of degree $\leq n$ determine the Pontryagin classes of $M$ as follows. The homomorphism $q_{1}^{*}: H^{\bullet}(M) \rightarrow H^{\bullet}\left(J^{1} \mathcal{M}_{M}\right)$ is an isomorphism, the inverse map being $\left(j^{1} g\right)^{*}$ for every $g \in \mathfrak{M e t} M$, and the Pontryagin classes of $M$ correspond - under this isomorphism - to the cohomology classes of the universal Pontryagin forms, as follows from the preceding theorem. However, a more remarkable result is the fact that there are non-zero universal Pontryagin forms of degree $>n$ (as $\operatorname{dim}\left(J^{1} \mathcal{M}_{M}\right)>n$ ), whereas the corresponding Pontryagin classes vanish for dimensional reasons. For example, for a surface the first 
universal Pontryagin form $p_{1}(\boldsymbol{\Omega}) \in \Omega^{4}\left(J^{1} \mathcal{M}_{M}\right)$ does not vanish. In fact, the study of this form is the principal goal of this paper.

Moreover, as the universal Levi-Civita connection $\boldsymbol{\omega}$ is $\mathcal{G}$-invariant, the BerlineVergne construction of equivariant characteristic classes (see $[3,4]$ ) provides canonical $\mathcal{G}$-equivariant extensions of the Pontryagin forms, which we call the equivariant Pontryagin forms $p_{k}\left(\boldsymbol{\Omega}^{\mathcal{G}}\right) \in \Omega_{\mathcal{G}}^{4 k}\left(J^{1} \mathcal{M}_{M}\right)$.

\section{Pre-symplectic Structure and Moment Map on $\mathfrak{M e t} M$}

According to [6], for an arbitrary fibred manifold $p: E \rightarrow M$, the differential forms on $J^{r} E$ of degree $\geq n$ determine differential forms on the space $\Gamma(M, E)$. By applying this construction to the bundle of Riemannian metrics, we obtain a map Im: $\Omega^{n+k}\left(J^{1} \mathcal{M}_{M}\right) \rightarrow \Omega^{k}(\operatorname{Met} M)$, given by

$$
\operatorname{Im}[\alpha]=\int_{M} \operatorname{ev}^{*} \alpha \in \Omega^{k}(\mathfrak{M e t} M)
$$

for every $\alpha \in \Omega^{n+k}\left(J^{1} \mathcal{M}_{M}\right)$, where ev: $M \times \mathfrak{M e t} M \rightarrow J^{1} \mathcal{M}_{M}$ is the evaluation map ev $(x, g)=j_{x}^{1} g$. The map Im commutes with the exterior differential and with the action of $\mathcal{G}^{+}$, and hence it transforms closed (resp. $\mathcal{G}^{+}$-invariant) forms into closed (resp. $\mathcal{G}^{+}$-invariant) forms. In addition, the map Im naturally extends to a map defined onto the spaces of $\mathcal{G}^{+}$-equivariant differential forms $\operatorname{Im}: \Omega_{\mathcal{G}^{+}}^{n+k}\left(J^{1} \mathcal{M}_{M}\right) \rightarrow \Omega_{\mathcal{G}^{+}}^{k}(\mathfrak{M e t} M)$, which commutes with the Cartan differential; for the details of this construction, we refer the reader to [6].

By applying Im to the equivariant Pontryagin forms of degree $4 k>n$, we obtain canonical closed $\mathcal{G}^{+}$-equivariant differential forms on $\operatorname{Met} M, \operatorname{Im}\left[p_{k}(\boldsymbol{\Omega})^{\mathfrak{M e t} M}\right] \in$ $\Omega_{\mathcal{G}^{+}}^{4 k-n}(\mathfrak{M e t} M)$.

From now on, we assume $n=\operatorname{dim} M=2$. As explained above, the first Pontryagin form $p_{1}(\boldsymbol{\Omega})=-\frac{1}{8 \pi^{2}} \operatorname{tr}(\boldsymbol{\Omega} \wedge \boldsymbol{\Omega}) \in \Omega^{4}\left(J^{1} \mathcal{M}_{M}\right)$, is closed and $\mathcal{G}^{+}$invariant. By applying the map Im to it, we obtain a closed $\mathcal{G}^{+}$-invariant twoform $\sigma=\operatorname{Im}\left[p_{1}(\boldsymbol{\Omega})\right] \in \Omega^{2}(\mathfrak{M e t} M)$, i.e., a $\mathcal{G}^{+}$-invariant pre-symplectic form on the space $\mathfrak{M e t} M$. Furthermore, by applying $\operatorname{Im}$ to the first equivariant Pontryagin form $p_{1}\left(\boldsymbol{\Omega}^{\mathcal{G}^{+}}\right) \in \Omega_{\mathcal{G}^{+}}^{4}\left(J^{1} \mathcal{M}_{M}\right)$ we obtain a canonical $\mathcal{G}^{+}$-equivariant extension of $\sigma, \sigma+\mu$, with $\mu: \mathfrak{X}(M) \times \mathbb{R} \rightarrow \Omega^{0}(\mathfrak{M e t} M)$. Due to the well-known relationship (e.g., see [2]) between equivariant extensions of a pre-symplectic form and moment maps, this equivariant extension determines a canonical moment map for the symplectic action of $\mathcal{G}^{+}$on $(\mathfrak{M e t} M, \sigma)$. Hence, the first universal Pontryagin form of a surface $M$ determines a canonical pre-symplectic form on $\mathfrak{M e t} M$, and 
the first equivariant Pontryagin form determines a canonical moment map for it. The explicit expressions of $\sigma$ and $\mu$ are given in the following

Theorem 3. For every $g \in \operatorname{Met} M, h, k \in \Gamma\left(M, S^{2} T^{*} M\right) \cong T_{g} \mathfrak{M e t} M$, $X \in \mathfrak{X}(M), t \in \mathbb{R}$ we have

$$
\begin{aligned}
\sigma_{g}(h, k)= & \frac{1}{4 \pi^{2}} \int_{M} \operatorname{tr}\left(\left(g^{-1} h \circ g^{-1} k\right)_{\mathrm{A}} \circ \Omega^{g}\right) \\
& +\frac{1}{4 \pi^{2}} \int_{M} \operatorname{tr}\left(\left(\dot{\nabla}^{g} h\right)_{\mathrm{A}} \wedge\left(\dot{\nabla}^{g} k\right)_{\mathrm{A}}\right) \\
\mu(X, t)_{g}= & \frac{1}{4 \pi^{2}} \int_{M} \operatorname{tr}\left(\nabla^{g} X \circ \Omega^{g}\right)
\end{aligned}
$$

where $\Omega^{g}$ is the curvature form of $g,\left(g^{-1} h\right)_{j}^{i}=g^{a i} h_{j a},\left(\dot{\nabla}^{g} h\right)_{A}$ denotes the skewsymmetric part of $\dot{\nabla}^{g} h$ and $\dot{\nabla}^{g} h \in \Omega^{1}(M, \operatorname{End} T M), \nabla^{g} X \in \Omega^{0}(M, \operatorname{End} T M)$ are given by

$$
\begin{aligned}
\left(\nabla^{g} X\right)_{j}^{i} & =\frac{\partial X^{i}}{\partial x^{j}}+\Gamma_{j k}^{i} X^{k} \\
\left(\dot{\nabla}^{g} h\right)_{j}^{i} & =g^{i b}\left(\frac{\partial h_{k b}}{\partial x^{j}}-h_{b a} \Gamma_{k j}^{a}-h_{k a} \Gamma_{b j}^{a}\right) \mathrm{d} x^{k} .
\end{aligned}
$$

Remark 4. More generally, if $M$ has dimension $n=4 k-2$ the $k$-th equivariant Pontryagin form determines a pre-symplectic structure and moment map on Met $M$, whose explicit expressions can be found in [8].

\section{Symplectic Reduction}

Next, we apply the Marsden-Weinstein procedure of symplectic reduction to the pre-symplectic manifold $(\mathfrak{M e t} M, \sigma)$ with respect to the moment map $\mu$.

Theorem 5. Let $g \in \mathfrak{M e t} M$ be a Riemaniann metric on the surface $M$. We have $\mu_{g}(X, t)=0$ for every $X \in \mathfrak{X}(M), t \in \mathbb{R}$ if and only if the scalar curvature $K^{g}$ of $g$, is constant. Hence $\mu^{-1}(0)$ is the space of metrics of constant curvature $\mathfrak{M e t}_{\mathrm{ct}} M$.

Assume, from now on, that the genus of $M$ is $\operatorname{gen}(M)>1$. By the Gauss-Bonnet theorem we have $\mathfrak{M e t}_{\text {ct }} M / \mathbb{R} \cong \mathfrak{M e t}_{-1} M$, the space of metrics of constant curvature -1 . Hence the Marsden-Weinstein quotient $\mu^{-1}(0) /\left(\operatorname{Diff}^{+} M \times \mathbb{R}\right) \cong$ Met $_{-1} M / \operatorname{Diff}^{+} M \cong \mathcal{M}(M)$ is the moduli space of complex structures on $M$. 
As the moduli space presents singularities due to the fact that the action of $\operatorname{Diff}^{+} M$ on $\mathfrak{M e t}_{-1} M$ is not free, it is customary to replace Diff ${ }^{+} M$ by the connected component of the identity Diffe $M \subset \operatorname{Diff} M$. The action of Diff ${ }^{e} M$ on $\mathfrak{M e t}_{-1} M$ is free, and the quotient space $\mathfrak{M e t}_{-1} M / \operatorname{Diff}^{e} M=\mathcal{T}(M)$ is the Teichmüller space of $M$.

The restriction of $\sigma$ to $\mathfrak{M e t}_{-1} M$ projects onto a canonical pre-symplectic form $\underline{\sigma}$ on $\mathcal{T}(M)$.

Moreover, the Teichmüller space is well known to be a Kähler manifold with the Weil-Petersson metric (e.g., see [10]), and hence we have two pre-symplectic forms on $\mathcal{T}(M)$ : The form $\underline{\sigma}$ obtained from the first Pontryagin form by means of symplectic reduction as defined above, and the Weil-Petersson symplectic form $\sigma_{\mathrm{WP}}$. The relationship between them is the following:

Theorem 6. We have $\underline{\sigma}=\frac{1}{2 \pi^{2}} \sigma_{\mathrm{WP}}$. Hence, the symplectic reduction of $(\mathfrak{M e t} M, \sigma)$ is $\left(\mathcal{T}(M), \frac{1}{2 \pi^{2}} \sigma_{\mathrm{WP}}\right)$.

Remark 7. Note that the preceding theorem provides an alternative proof of the fact that the Weil-Petersson metric on $\mathcal{T}(M)$ is Kähler, as we know that $\sigma$ is closed by its very definition, and hence $\sigma_{\mathrm{WP}}$ is also closed.

\section{Acknowledgements}

This work is supported by the Ministerio de Educación y Ciencia of Spain, under grant \# MTM2005-00173.

\section{References}

[1] Atiyah M. and Bott R., The Yang-Mills equations over Riemann surfaces, Phil. Trans. R. Soc. Lond. A 308 (1982) 523-615.

[2] Atiyah M. and Bott R., The Moment Map and Equivariant Cohomology, Topology 23 (1984) 1-28.

[3] Berline N. and Vergne M., Classes caractéristiques équivariantes. Formules de localisation en cohomologie équivariante, C. R. Acad. Sci. Paris 295 (1982) 539-541.

[4] Berline N. and Vergne M., Zéros d'un champ de vecteurs et classes caractéristiques équivariantes, Duke Math. J. 50 (1983) 539-549. 
[5] Epstein D., Natural Tensors on Riemannian Manifolds, J. Diff. Geom. 10 (1975) 631-645.

[6] Pérez R., Equivariant Characteristic Forms in the Bundle of Connections, J. Geom. Phys. 54 (2005) 197-212

[7] Pérez R. and Masqué J., Natural Connections on the Bundle of Riemannian Metrics, preprint (arXiv: math.DG/0507075).

[8] Pérez R. and Masqué J., Pontryagin Forms on (4k-2)-Manifolds and Symplectic Sstructures on the Spaces of Riemannian Metrics, preprint (arXiv: math.DG/0507076).

[9] Kobayashi S. and Nomizu K., Foundations of Differential Geometry, John Wiley \& Sons, New York, Volume I, 1963; Volume II, 1969.

[10] Tromba A., Teichmüller Theory in Riemannian Geometry, Birkhäuser, Basel, 1992.

Roberto Ferreiro Pérez

Departamento Economía Financiera y Contabilidad I

Facultad de Ciencias Económicas y

Empresariales, UCM

Campus de Somosaguas

28223-Pozuelo de Alarcón, SPAIN

E-mail address: roferreirodccee.ucm.es

Jaime Muñoz Masqué

Insituto de Física Aplicada, CSIC

C/ Serrano 144, 28006-Madrid, SPAIN

E-mail address: jaimediec.csic.es 